\title{
Lesões medulares traumáticas agudas sem alterações radiológicas relacionadas com 0
}

\section{trauma}

Francisco Sérgio C. Barros Leal, Antônio Celso A. Guimarães, Roberto R. Franco, José Carlos E. Veiga

Serviço de Neurocirurgia dos Hospitais das Clínicas e Universitário da Universidade de Taubaté (Unitau)

\section{RESUMO}

As lesões medulares traumáticas sem alterações radiológicas relacionadas com o trauma, como o próprio nome sugere, constituem uma entidade na qual há sinais clínicos de lesão medular na ausência de anomalias radiológicas próprias do traumatismo (radiografia simples e tomografia computadorizada). Contudo, podem estar associadas a outras alterações (espondilose, canal estreito, espondilite anquilosante, hérnias de disco, etc.). Caso a ressonância magnética fosse incluída na investigação, haveria uma redução significativa na incidência dessas lesões e dificilmente deixariam de ser diagnosticadas. Foram estudados 14 pacientes com idades entre 21 e 70 anos admitidos em nosso serviço, no período de 1989 a 1997, submetidos a estudo radiográfico simples (incluindo estudo dinâmico) e/ou tomografia computadorizada da coluna vertebral cervical. Em todos os casos, foram descartadas alterações radiológicas relacionadas com o trauma.

O sexo masculino predominou (13 casos) e houve uma preponderância do grupo etário entre a quinta e sétima décadas (11 casos). Todos tinham disfunções referentes à medula cervical, em algum momento: dois apresentavam tetraparesia transitória (concussão medular), nove apresentavam síndrome centromedular, um tinha tetraparesia de predomínio crural e, dois, tetraplegia flácida. O mecanismo do trauma foi hiperextensão cervical em 13 pacientes. Espondilose cervical foi observada em 13 casos. O diâmetro do canal cervical foi: inferior a $13 \mathrm{~mm}$, em um paciente; entre $13 \mathrm{~mm}$ e $14 \mathrm{~mm}$, em quatro; $15 \mathrm{~mm}$ e $16 \mathrm{~mm}$, em três; $17 \mathrm{~mm}$ e $18 \mathrm{~mm}$, em dois; e superior a $18 \mathrm{~mm}$, em um. Hérnia de disco traumática ocorreu em um caso. Três doentes morreram.

$\mathrm{Na}$ nossa casuística, as lesões medulares traumáticas sem alterações radiológicas relacionadas com o trauma foram mais comuns em homens, com mais de 40 anos, com estreitamento do canal vertebral, submetidos à hiperextensão cervical aguda. O prognóstico parece estar relacionado com a gravidade do quadro clínico inicial e as alterações encontradas na ressonância magnética.

A terminologia "lesões medulares traumáticas sem alterações radiológicas relacionadas com o trauma" parece-nos inadequada e deve ser revista.

\section{PALAVRAS-CHAVE}

Lesão medular cervical traumática. Traumatismo raquimedular.

\section{ABSTRACT}

Spinal cord injuries without radiological evidence of trauma Spinal cord injuries without radiological evidence of trauma (SCIWORET), as suggested by its terminology, is a condition where there are clinical signs of spinal cord dysfunction in the absence of characteristic radiological abnormalities (plain radiographs and computerized tomography), although may be associated with other anomalies (spondylosis, narrowing of cervical canal, ankylosing spondylitis, disc herniation, etc.). If magnetic resonance imaging (MRI) were used in such cases traumatic abnormalities would hardly be misidentified.

We studied 14 patients aged from 21 to 70 years admitted in the neurosurgical department between 1989 and 1997, submitted to plain radiographs (with flexion and extension views) and/or computerized tomography of the cervical spine. There was no radiological evidence of trauma in any of the cases.

There were thirteen male patients and one female. All had cervical cord dysfunction: two presented with transient tetraparesis (spinal cord concussion), nine with central spinal cord lesions, one patient with tetraparesia predominant 
on lower limbs and two with flaccid tetraplegia. Acute hyperextension of the cervical spine was the responsible mechanism in thirteen patients. Cervical spondylosis was observed in thirteen patients. The diameter of the cervical canal was: lower than $13 \mathrm{~mm}$ in one patient, between $13 \mathrm{~mm}$ and $14 \mathrm{~mm}$ in four, 15 and $16 \mathrm{~mm}$ in three, $17 \mathrm{~mm}$ and $18 \mathrm{~mm}$ in two and greater than $18 \mathrm{~mm}$ in one. Traumatic disk herniation was found in one patient. Three patients died. In our series acute traumatic spinal cord injuries without radiological evidence of trauma were prevalent in males over 40 years of age with narrowing of the cervical canal and submitted to acute cervical hyperextension. The prognosis seems to be related to the severity of the initial clinical findings and to the abnormalities found in the magnetic ressonance imaging.

The term "spine cord injury without radiological evidence of trauma" seems inappropriate and should be reviewed.

\section{KEYWORDS}

SCIWORA. SCIWORET. Spinal cord injury. Spinal trauma.

\section{Introdução}

As lesões medulares traumáticas sem alterações radiológicas relacionadas com o trauma, como o próprio nome indica, constituem uma entidade na qual há sinais clínicos de disfunção e/ou lesão medular na ausência de alterações radiológicas próprias do traumatismo (fraturas ou luxações). Entretanto, outras alterações freqüentemente estão relacionadas a essas disfunções e/ou lesões (espondilose, canal estreito, embolia do núcleo pulposo, espondilite anquilosante, hérnias de disco, etc.) ${ }^{12}$. É possível reconhecer três grupos diferentes de pacientes: 1) o grupo no qual não são observadas quaisquer alterações radiológicas, denominado "Spinal cord injury without radiologic abnormality" (SCIWORA); 2) o grupo no qual não são observadas alterações radiológicas relacionadas com o traumatismo, mas que apresenta as alterações acima descritas, denominado Spinal cord injury without radiologic evidence of trauma (SCIWORET); 3) um grupo raro de pacientes que sofrem disfunção e/ou lesão medular secundária ao traumatismo distante da coluna vertebral (geralmente associada à hipotensão prolongada, choque e lesões de artérias nutridoras da medula $)^{12}$.

Um subgrupo de pacientes com alterações de menor gravidade e reversíveis tem sido descrito (concussões medulares) $)^{3,12}$.

SCIWORA é mais freqüentemente observado na idade pediátrica, apesar de ocorrer também na idade adulta. O SCIWORET ocorre predominantemente em adultos. $\mathrm{O}$ terceiro grupo não parece ter relação com a idade ${ }^{12}$.

Por definição, as alterações radiológicas referemse às obtidas nas radiografias simples (RX) e tomografia computadorizada (TC). Antes da utilização da TC no estudo dos traumatismos raquimedulares, a incidência de SCIWORET, em adultos, era de $14 \%$; com o advento dessa, esse número caiu para apenas $5 \%{ }^{11}$. O estudo por ressonância magnética $(\mathrm{RM})$ implica na redução ainda maior de disfunções e/ou lesões medulares traumáticas sem as alterações de imagem correspondentes.

As alterações observadas na RM são extraneurais (lesões de ligamentos e disco) ou neurais (hemorragia e edema medular), havendo nítida correlação das alterações com o prognóstico ${ }^{2,7}$.

Do ponto de vista fisiopatológico, as lesões nos adultos ocorrem, provavelmente, em decorrência de hiperextensão à qual foi submetida a coluna com alterações espondilóticas ou com canal estreitado, levando, geralmente, a uma síndrome centromedular, inclusive nas "lesões menores" ", $7,8,13,14$. Segundo Taylor e Blackwood $^{14}$, a hiperextensão forçada provocaria ruptura do ligamento longitudinal anterior e listese temporária da vértebra superior. Em seguida, ocorreria a redução espontânea devido à ação da musculatura espinhal, o que explica a aparência normal ao $\mathrm{RX}^{14}$.

Em crianças, uma série de particularidades anatômicas seriam responsáveis por uma maior susceptibilidade à lesão e/ou à disfunção medular: superfícies articulares mais horizontalizadas, configuração do corpo vertebral facilitando o escorregamento de um corpo vertebral sobre o outro, maior elasticidade dos ligamentos e das cápsulas articulares, musculatura cervical pouco desenvolvida, peso desproporcional da cabeça em relação ao restante do corpo (corresponde a $1 / 4$ do peso corporal e, proporcionalmente, pesa quatro vezes mais que no adulto). Tais peculiaridades, associadas à ausência de uma angulação uniforme, de lordose e de curvas de flexão cervicais, tornam freqüentes a ocorrência de luxações seguidas de redução espontânea na infância ${ }^{2,9,10,15,16,18}$.

As lesões do tipo SCIWORA e SCIWORET são, portanto, causadas por uma sobrecarga nos discos e ligamentos sem a evidência radiológica de instabilidade ${ }^{5}$.

Este trabalho tem o objetivo de estudar os fatores envolvidos na gênese das lesões medulares traumáticas agudas sem alterações radiológicas relacionadas com o trauma e fazer uma análise crítica da terminologia utilizada.

\section{Casuística e métodos}

Foram estudados, retrospectivamente, 14 pacientes com traumatismo raquimedular, admitidos em nosso serviço, entre 1989 e 1997, portadores de déficit neurológico (permanente ou transitório), nos quais o RX (mesmo após estudo dinâmico do segmento 
afetado) e a TC não mostravam alterações relacionadas com o traumatismo (fraturas ou luxações). Quatro pacientes foram submetidos à perimielografia e/ou mielotomografia e, quatro outros, à RM.

Os doentes foram analisados quanto à idade, ao sexo, ao mecanismo do trauma, ao quadro clínico, à presença de espondilose, ao diâmetro do canal e à evolução (Tabela 1). Foram revistos os exames de 11 desses pacientes, para averiguar a presença de alterações não relacionadas com o traumatismo e medir o diâmetro do canal vertebral nestes. Nos outros três pacientes, os dados foram obtidos pelas anotações de prontuário.

Em cinco casos, foi administrada metilprednisolona endovenosa, todos admitidos até oito horas após o trauma ( $30 \mathrm{mg} / \mathrm{kg}$ como dose de ataque $+5,4 \mathrm{mg} / \mathrm{kg} / \mathrm{hora}$ nas 23 horas subseqüentes, em infusão contínua). Todos os pacientes internados receberam heparina profilaticamente por via subcutânea. Apenas um paciente foi operado (cirurgia de Smith-Robinson), sendo os demais tratados clinicamente apenas com colar cervical.

\section{Resultados}

Quanto à faixa etária, um paciente tinha entre $21 \mathrm{e}$ 30 anos, dois entre 31 e 40, quatro entre 41 e 50, três entre 51 e 60 e quatro entre 61 e 70 (Figura 1). Treze pacientes eram do sexo masculino.

Quanto ao mecanismo do trauma, a causa foi hiperextensão forçada da coluna cervical em 13 casos, baseada no tipo de traumatismo sofrido e evidência clínica e/ou radiológica de traumatismo na porção anterior ou ântero-lateral do crânio, incluindo traumatismos faciais (Tabela 1).

Dois pacientes tiveram tetraparesia transitória e, portanto, foram classificados, no quadro clínico, como concussões medulares. Dos doze restantes, nove apresentaram síndrome centromedular, um apresentava tetraparesia de predomínio crural e, dois outros, tetraplegia. A síndrome centromedular estava associada a parestesias em ambas as mãos (burning hands syndrome), em dois, um dos quais apresentava também síndrome de Brown-Séquard (Tabela 1). A associação com alcoolismo ocorreu em três casos.

Foram observadas alterações radiológicas compatíveis com espondilose cervical em 13 pacientes. A medida do canal vertebral realizada, em 11 casos, revelou: um paciente com diâmetro inferior a $13 \mathrm{~mm}$, quatro com diâmetro entre $13 \mathrm{~mm}$ e $14 \mathrm{~mm}$, três entre $15 \mathrm{~mm}$ e $16 \mathrm{~mm}$, dois com diâmetros entre $17 \mathrm{~mm}$ e $18 \mathrm{~mm}$, e apenas um com diâmetro superior a $18 \mathrm{~mm}$, mas que apresentava fusão congênita dos corpos vertebrais de $\mathrm{C} 4$ e $\mathrm{C} 5$. Em 73\% desses, a medida do canal vertebral variou entre $13 \mathrm{~mm}$ e $16 \mathrm{~mm}$ (Figuras 2 e 3). O achado de hérnia de disco cervical traumática ocorreu em um caso.

Os dois pacientes admitidos com concussão medular receberam alta, entre 48 e 72 horas desde a internação, e assintomáticos. Os tetraparéticos (incluindo os portadores de síndrome centromedular) tiveram um grau variável de melhora clínica, inversamente proporcional à gravidade da lesão inicial. Os dois pacientes tetraplégicos e um tetraparético evoluíram para óbito perfazendo uma mortalidade de $21,4 \%$ (Figura 4).

\begin{tabular}{|c|c|c|c|c|c|c|c|}
\hline \multicolumn{8}{|c|}{$\begin{array}{l}\text { Tabela } 1 \\
\text { ral dos pacientes }(n=14)\end{array}$} \\
\hline Paciente & Idade & Sexo & Mecanismo & Quadro clínico & Espondilose & Canal & Evolução \\
\hline 1 & 38 & $\mathrm{~m}$ & hiperextensão & SCM & $\operatorname{sim}$ & $14 \mathrm{~mm}$ & melhora \\
\hline 2 & 48 & $\mathrm{~m}$ & hiperextensão & TPL & $\operatorname{sim}$ & $15 \mathrm{~mm}$ & óbito \\
\hline 3 & 70 & $\mathrm{~m}$ & hiperextensão & $\mathrm{SCM}$ & $\operatorname{sim}$ & $18 \mathrm{~mm}$ & melhora \\
\hline 4 & 51 & $\mathrm{~m}$ & hiperextensão & DT & $\operatorname{sim}$ & $\mathrm{nm}$ & cura \\
\hline 5 & 59 & $\mathrm{~m}$ & hiperextensão & SCM & $\operatorname{sim}$ & $14 \mathrm{~mm}$ & melhora \\
\hline 6 & 34 & $\mathrm{~m}$ & hiperextensão & $\mathrm{SCM}+\mathrm{BHS}+\mathrm{SBS}$ & $\operatorname{sim}$ & $14 \mathrm{~mm}$ & melhora \\
\hline 7 & 48 & $\mathrm{~m}$ & hiperextensão & $\mathrm{SCM}$ & $\operatorname{sim}$ & $16 \mathrm{~mm}$ & melhora \\
\hline 8 & 58 & $\mathrm{~m}$ & hiperextensão & $\mathrm{SCM}$ & $\operatorname{sim}$ & $\mathrm{nm}$ & melhora \\
\hline 9 & 21 & $\mathrm{~m}$ & hiperextensão & DT & não & $\mathrm{nm}$ & cura \\
\hline 10 & 66 & $\mathrm{~m}$ & hiperextensão & $\mathrm{SCM}+\mathrm{BHS}$ & $\operatorname{sim}$ & $15 \mathrm{~mm}$ & melhora \\
\hline 11 & 68 & $\mathrm{f}$ & hiperflexão & TPL & $\operatorname{sim}$ & $19 \mathrm{~mm}$ & óbito \\
\hline 12 & 50 & $\mathrm{~m}$ & hiperextensão & $\mathrm{SCM}$ & $\operatorname{sim}$ & $17 \mathrm{~mm}$ & melhora \\
\hline 13 & 43 & $\mathrm{~m}$ & hiperextensão & SCM & $\operatorname{sim}$ & $12 \mathrm{~mm}$ & melhora \\
\hline 14 & 70 & $\mathrm{~m}$ & hiperextensão & TPR & $\operatorname{sim}$ & $13 \mathrm{~mm}$ & óbito \\
\hline
\end{tabular}

$\mathrm{SCM}=$ síndrome centromedular; BHS = burning hands syndrome; $\mathrm{TPR}=$ tetraparesia; $\mathrm{TPL}=$ tetraplegia; $\mathrm{DT}=$ déficit transitório; $\mathrm{SBS}=$ síndrome de Brown-Sèquard $; \mathrm{nm}=$ não medido $; \mathrm{m}=$ masculino $; \mathrm{f}=$ feminino . 


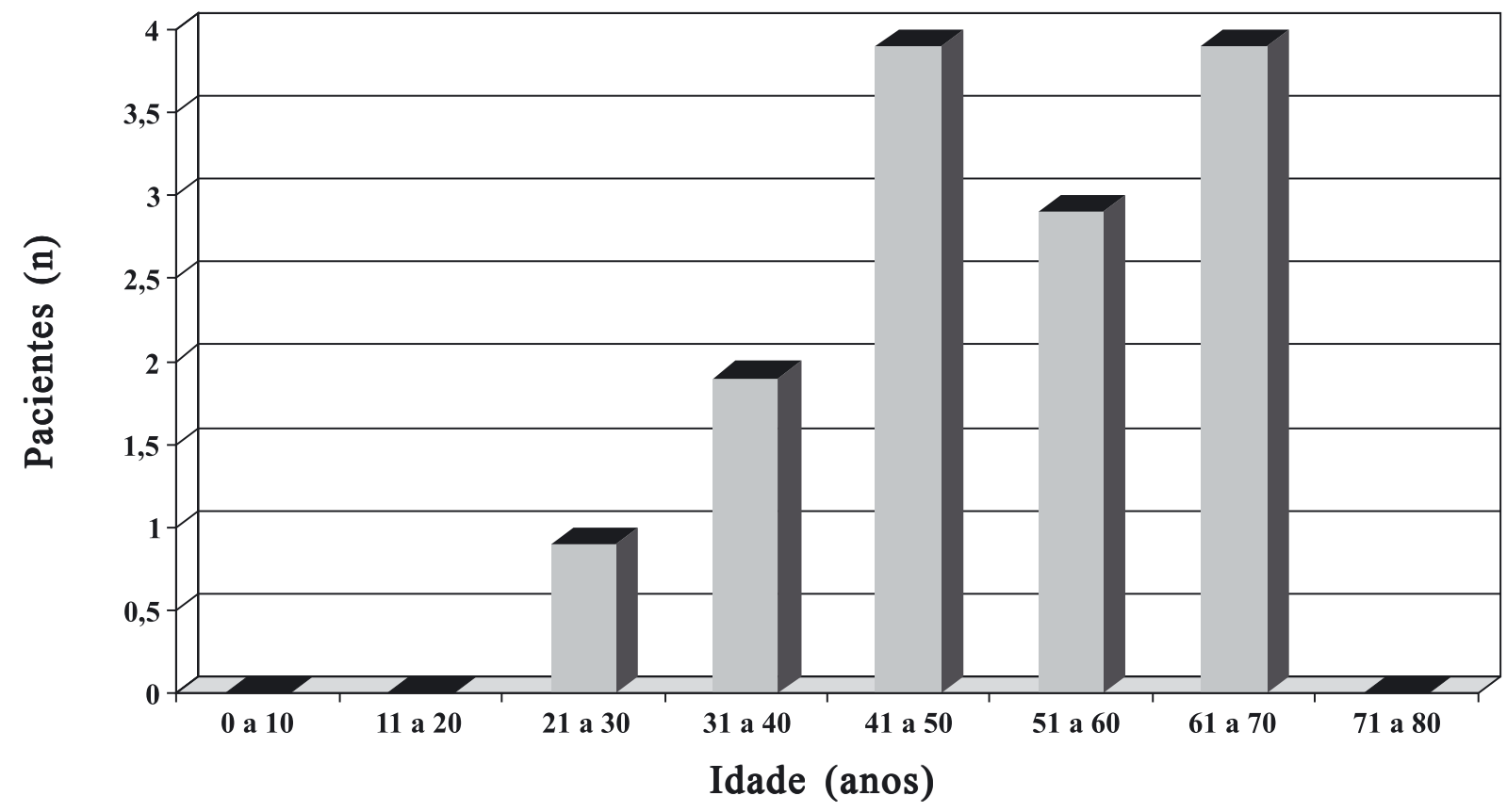

Figura 1 - Distribuição dos pacientes quanto à idade $(n=14)$

\section{Discussão}

Lesões medulares traumáticas sem alterações radiológicas relacionadas com o trauma são conhecidas de longa data ${ }^{1,6,7,8}$. Bennett, em 1950, (apud Schneider e cols. ${ }^{7}$ ) foi o primeiro autor a fazer uma observação anatômica de que a hematomielia poderia ocorrer sem a presença de lesões ósseas, e Schneider ${ }^{7}$, em 1954, descreveu, pela primeira vez, a síndrome centromedular. A associação de lesões centromedulares dar-se-ia devido a uma compressão ântero-posterior da medula exercida tanto por osteófitos, como por um preguea-

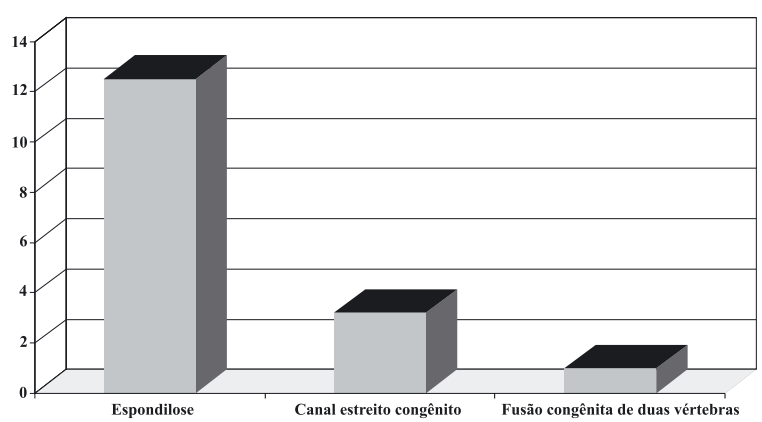

Figura 2 - Distribuição dos pacientes de acordo com as alterações radiológicas encontradas $(n=14)$ mento anormal do ligamento amarelo hipertrofiado. Tais alterações, aliadas a uma lordose cervical compensatória secundária a uma cifose torácica senil, causariam diminuição do diâmetro do canal vertebral em cerca de $15 \%{ }^{1,4,7,8}$. Na hiperextensão forçada, pode ocorrer uma diminuição do diâmetro do canal de até $50 \%{ }^{1}$.

Os 14 casos aqui apresentados são compostos de adultos com idades entre 21 e 70 anos, portadores de estreitamento de canal vertebral cervical, seja por espondilose ou por estenose congênita.

Nossa casuística caberia melhor no conceito das lesões tipo SCIWORET, já que nas lesões do tipo

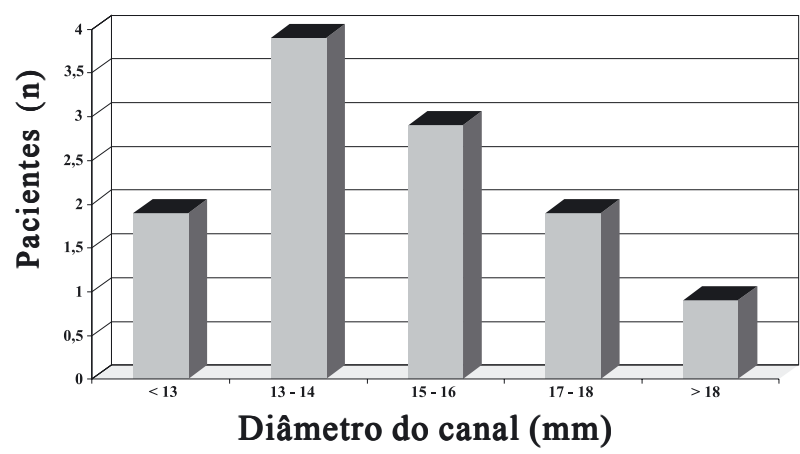

Figura 3 - Distribuição dos pacientes de acordo com o diâmetro do canal $(n=14)$ 


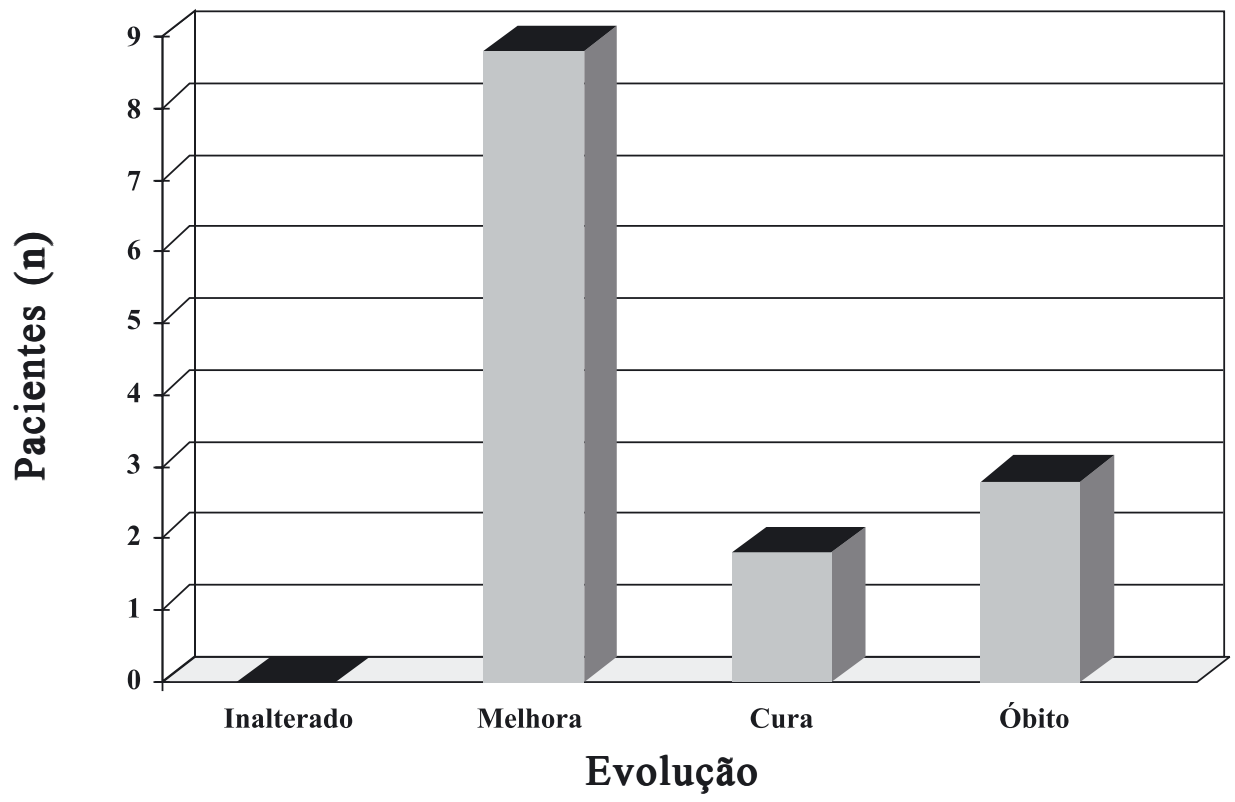

Figura 4 - Evolução dos pacientes $(n=14)$

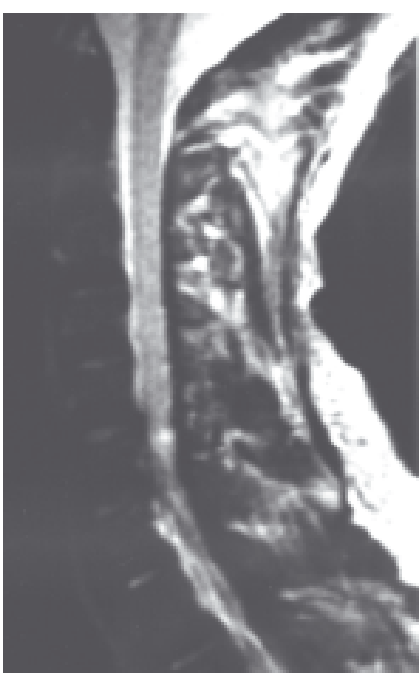

Figura 5 - Paciente $n^{0}$ 6: 34 anos, sofreu traumatismo cervical em hiperextensão por acidente de moto. A RM, em T2, mostra uma associação de espondilose com estreitamento congênito do canal vertebral. Observe a presença de alteração do sinal intramedular em C5/C6 decorrente de mielomalacia.

SCIWORA normalmente não existe alteração radiográfica alguma e são mais comuns na infância. Tais denominações são, no mínimo, inadequadas, em virtude da existência, na grande maioria das vezes, de alterações na RM (tanto nas SCIWORA, quanto nas SCIWORET) ${ }^{12}$. As figuras 5 e 6 são exemplos clássicos de SCIWORET.

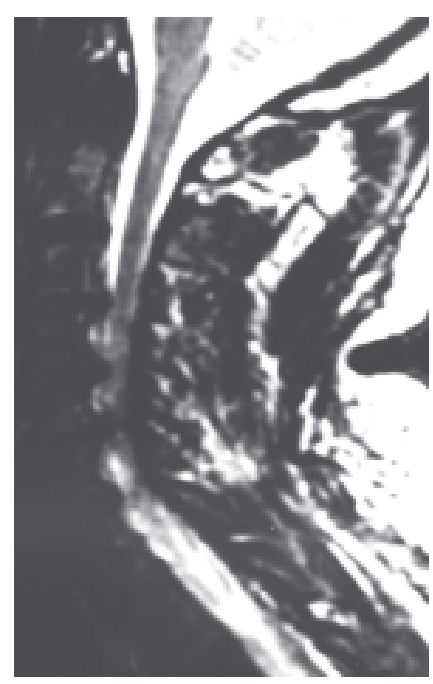

Figura 6 - Paciente no 7: 48 anos, vítima de traumatismo cervical em hiperextensão por coice de cavalo. A RM, em

$T 2$, mostra alterações espondilóticas avançadas em múltiplos níveis e alteração do sinal intramedular por mielomalacia em C6.

Em nosso estudo, não observamos nenhum paciente com lesões medulares secundárias a traumatismos fora da coluna vertebral (associados com hipotensão prolongada, choque e lesões de artérias nutridoras da medula), como o descrito por Tator ${ }^{12}$.

Barnes, em 1948, (apud Hughes e Brownell ${ }^{6}$ ) descreveu 22 pacientes com traumatismo raquimedular 
cervical e identificou dois grupos distintos quanto ao mecanismo de lesão. O primeiro grupo é composto de pacientes que sofreram traumatismos em hiperflexão, geralmente portadores de lesões do tipo fratura e/ou luxações e herniações discais agudas, com idade média inferior a 50 anos. O segundo consiste em pacientes com idades superiores a 57 anos, normalmente com traumatismos em hiperextensão, sem evidências radiológicas de traumatismo (apenas sinais de espondilose).

Neste estudo, todos os pacientes apresentam evidências radiológicas de espondilose cervical e/ou estenose de canal. Um desses (paciente 11), apesar de uma espondilose severa, apresenta canal com diâmetro de 19 mm, associado à fusão congênita de dois corpos vertebrais (também descrito como associado a esse tipo de lesão) ${ }^{6}$.

\section{Conclusões}

A análise do grupo estudado está de acordo com a literatura pertinente e permite concluir que:

1. Traumatismos cervicais associados a lesões medulares sem alterações radiográficas são uma entidade que ocorre, predominantemente, em pessoas idosas, do sexo masculino, submetidos à hiperextensão forçada da coluna cervical.

2. Traumatismos cervicais associados a lesões medulares sem alterações radiográficas podem acometer pessoas jovens portadoras de estenose de canal vertebral. As lesões são mais freqüentes quanto mais estreito for o canal e podem, eventualmente, estar associadas à fusão congênita de corpos vertebrais.

3. O prognóstico dessas lesões parece estar associado à gravidade do quadro clínico e às alterações encontradas na ressonância magnética. As concussões medulares possuem um excelente prognóstico, enquanto as lesões medulares completas têm uma mortalidade alta. Os doentes com lesões medulares incompletas parecem ter sempre algum grau de recuperação funcional, inversamente proporcional à lesão inicial.

4. A melhor maneira de prevenir essas lesões é o diagnóstico precoce das alterações sabidamente associadas (estenose de canal e espondilose cervical) e orientação adequada.

5. Os termos SCIWORA e SCIWORET não são apropriados porque, com o uso cada vez maior da ressonância magnética nos traumatismos raquimedulares, as lesões relacionadas com o traumatismo dificilmente deixarão de ser diagnosticadas.

\section{Referências}

1. ALEXANDER Jr, E DAVIS $\mathrm{CH} J \mathrm{~J}$, FIELD $\mathrm{CH}$ : Hyperextension injuries of the cervical spine. Arch Neurol Psychiatry 79:146-50, 1958.

2. BAILEY DK: The normal cervical spine in infants and children. Radiology 59:712-9, 1952.

3. EICHLER ME, VOLLMER DG: Cervical spine trauma. In: Youmans Jr (ed): Neurological Surgery. Ed 4. Philadelphia, Saunders, 1996.

4. FEHLING MG, ARIAS JM: Nonsurgical management of spinal cord injuries. In: Tindall GT, Cooper PR, Barrow DL (eds): The practice of neurosurgery. Ed 1. Baltimore, Williams and Wilkins, 1996, vol 2, pp 16731685.

5. GRABB PA, PANG D: Magnetic resonance imaging in the evaluation of the spinal cord injury without radiological abnormality in children. Clinical study. Neurosurgery 35:406-14, 1994.

6. HUGHES JT, BROWNELL B: Spinal cord damage from hyperextension injury in cervical spondylosis. Lancet 1:687-90, 1963.

7. SCHNEIDER RC, CHERRY G, PATEK H: The syndrome of acute central cervical spine cord injury: with special reference to the mechanisms involved in hyperextension injuries of cervical spine. J Neurosurg 11:546-77, 1954.

8. SCHNEIDER RC, THOMPPSON JM, BEBIN J: The syndrome of acute central cervical spinal cord injury. J Neurol Neurosurg Psychiatry 21:216-27, 1958.

9. SHERK HH, SCHUT L, LANE JM: Fractures and dislocations of the cervical spine in children. Orthop Clin North Am 7:593-604, 1976.

10. SULLIVAN CR, BRUWER AJ, HARRIS LE: Hypermobility of the cervical spine in children: a pitfall in the diagnosis of cervical dislocation. Am J Surg 95:636-640, 1958.

11. TATOR $\mathrm{CH}$ : Spine-spinal cord relationships in spinal cord trauma. Clin Neurosurg 30:479-94, 1983.

12. TATOR $\mathrm{CH}$ : Spinal cord syndromes with physiological and anatomic correlations. In: Menezes AH, Sontag VKH: Principles of spinal surgery. Ed 1. McGraw-Hill, 1996, vol 2, pp 785-99.

13. TAYLOR AR: The mechanism of injury to the spinal cord in the neck without damage to the vertebral column. J Bone Joint Surg [Br] 33:543-7, 1951.

14. TAYLOR AR, BLACKWOOD W: Paraplegia in cervical injuries with normal radiographic appearance. J Bone Joint Surg [Br] 30:245-8, 1948.

15. TOWBIN A: Spinal injury related to the syndrome of sudden death ("crib-death") in infants. Am J Clin Pathol 49:562-7, 1968.

16. TOWNSEND Jr EH, ROWE ML: Mobility of the upper cervical spine in health and disease. Pediatrics 10:567-73, 1952.

Original recebido em janeiro de 1999

Aceito para publicação em setembro de 1999

\section{Endereço para correspondência:}

Francisco Sérgio C. Barros Leal

Travessa dos Rubis, 37 - Residencial Eldorado - Tremembé

CEP 12120-000 - São Paulo - SP 